\title{
Carrier-Envelope Offset Phase-Locking With Attosecond Timing Jitter
}

\author{
Florian W. Helbing, Günter Steinmeyer, Member, IEEE, and Ursula Keller, Senior Member, IEEE
}

\begin{abstract}
Inside a femtosecond laser oscillator, no coupling mechanism between the propagation speeds of the carrier and the pulse envelope exists. Therefore, the relative delay between carrier and envelope of a femtosecond oscillator will exhibit irregular fluctuations unless this jitter is actively suppressed. Both intensity and beam pointing fluctuations in the laser can introduce carrier-envelope phase changes. Based on our analysis, we are capable of reducing or avoiding certain mechanisms by proper design of the laser cavity. We use such an optimized cavity to stabilize the carrier envelope-phase to an external reference oscillator with a long-term residual jitter corresponding to only 10 attoseconds in a $(100 \mathrm{kHz}-0.01 \mathrm{~Hz})$ bandwidth. This is the smallest long-term timing jitter of a femtosecond laser oscillator demonstrated to date. However, it is important to note that this stabilization was obtained with an $f$-to- $f$ heterodyne technique using additional external spectral broadening in a microstructure fiber which introduces additional carrier-envelope phase noise. We present a direct heterodyne measurement of this additional carrier-envelope phase noise due to the continuum generation process.
\end{abstract}

Index Terms-Mode-locked lasers, optical Kerr effect, phase jitter, phase synchronization, ultrafast optics.

\section{INTRODUCTION}

$\mathbf{P}$ ROGRESS in ultrashort pulse generation [1] has reached a level where the slowly varying envelope approximation starts to fail. Pulse durations of Ti:sapphire laser oscillators have reached around $5 \mathrm{fs}$, which is so short that only about two optical cycles of the underlying electric field fit into the full-width half maximum (FWHM) of the pulse envelope (Fig. 1) [2], [3]. Similar pulse durations but much more energy can be obtained from hollow fiber pulse compression [4] and synchronously pumped optical parametric oscillators [5]. More recently, even shorter pulses of $3.8 \mathrm{fs}$ have been achieved with adaptive pulse compression of a cascaded hollow fiber supercontinuum [6]. These are currently among the shortest pulses generated in the visible and near-infrared spectral regime. For such short pulses the maximum electric field strength depends strongly on the exact position of the electric field with regards to the pulse envelope, i.e., the carrier envelope offset (CEO) [7]. In passively modelocked

Manuscript received December 23, 2002; revised May 14, 2003. This work was supported by ETH Zürich and by the Swiss National Center of competence in research (NCCR) in quantum photonics.

F. W. Helbing and U. Keller are with the Institute of Quantum Electronics, Swiss Federal Institute of Technology, CH-8093 Zürich, Switzerland (e-mail: helbing@phys.ethz.ch).

G. Steinmeyer is with the Max-Born-Institut für Nichtlineare Optik und Kurzzeitspektroskopie, D-12489 Berlin, Germany (e-mail: steinmey@mbiberlin.de).

Digital Object Identifier 10.1109/JSTQE.2003.819104

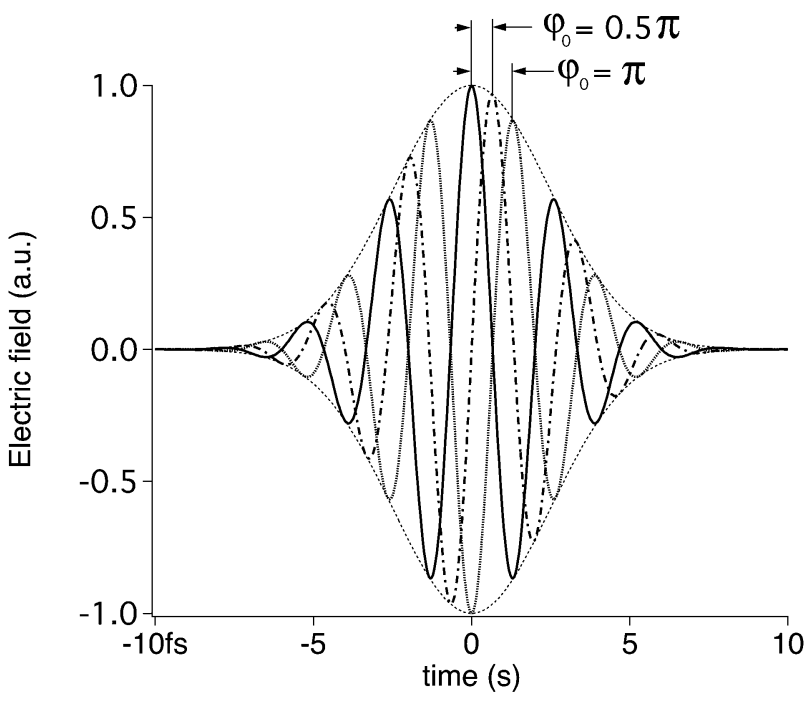

Fig. 1. Electric field structure of a few-cycle pulse for different values of the carrier-envelope-offset phase $\varphi_{0}$ of 0 (solid line), $0.5 \pi$ (dashed-dotted line), and $\pi$ (dotted line). Envelope $\pm A(t)$ is also shown.

lasers this carrier envelope offset is a freely varying parameter because the steady-state boundary condition only requires that the pulse envelope is the same after one round trip. Therefore, the CEO phase may exhibit large fluctuations, even when all other laser parameters are stabilized. We have discussed the physical origin of these fluctuations before [8], [9] and will review them later in this paper. Because nonlinear laser-matter interaction depends strongly on the strength of the electric field, these CEO fluctuations cause strong signal fluctuations in nonlinear experiments such as high harmonic generation [10], attosecond pulse generation [11], photoelectron emission [12], etc.

Different techniques have been proposed to measure the CEO frequency in the time domain [13] and in the frequency domain [7]. The frequency domain technique is much more sensitive and is generally the technique that is being used today. Using the $f$-to- $2 f$ heterodyne technique with additional external spectral broadening [14], [15] we achieved a long-term CEO stabilization with residual 10 -attosecond timing jitter which corresponds to $0.025 \mathrm{rad}$ rms CEO phase noise in a $(0.01 \mathrm{~Hz}-100 \mathrm{kHz})$ bandwidth. The $f$-to- $2 f$ interference technique requires an octave-spanning spectrum. So far, all attempts to utilize an octave spanning spectrum directly from a laser source for measuring the CEO frequency with an $f$-to- $2 f$ scheme have only reached unsatisfactory control of the CEO frequency, which was mainly caused by poor signal strength [16]. Recently, a $2 f$-to- $3 f$ scheme with an improved signal to noise ratio (SNR) and CEO 
phase-locking capability was presented [17]. However, the published SNR is inferior to those routinely achieved with measurement setups employing externally broadened spectra. Therefore, most experiments employ additional spectral broadening, e.g., in an external microstructure fiber. The continuum generation process with its strong nonlinearity, however, introduces additional CEO noise. It is important to note that the CEO stabilization is achieved for the pulses after the microstructure fiber, which means that the pulses directly from the Ti:sapphire laser will exhibit excess CEO phase noise even with a perfectly working CEO stabilization. In our experiments, we observed typical phase drift values of about $1.2 \mathrm{rad} / \mathrm{mW}$ oscillator power fluctuations, as will be discussed in more detail later in this paper.

\section{CEO FREQUENCY}

In the following, we restrict our discussion to the frequency domain technique [7]. In the time domain the passively modelocked pulse from a Ti:sapphire laser can be described as

$$
E(t)=A(t) \exp \left(i \omega_{c} t+i \varphi_{0}(t)\right)
$$

where $E(t)$ is the electric field wave packet, $\omega_{c}$ is the (angular) carrier frequency which is the center of gravity of the mode-locked spectrum, $A(t)$ the pulse envelope, and $\varphi_{0}(t)$ the absolute phase of the pulse (Fig. 1). Inside the cavity the carrier propagates with phase velocity $v_{p}$, whereas the envelope propagates with the group velocity $v_{g}$.

For $v_{g}=v_{p}$, both phases evolve equally with time, i.e., $\varphi_{0}(t)=$ const, and the electric field pattern is repeated at the pulse repetition period $T_{R}$

$$
E_{\text {train }}(t)=A(t) \exp \left(i \omega_{c} t+i \varphi_{0}(t)\right) \otimes \sum_{m=-\infty}^{+\infty} \delta\left(t-m T_{R}\right) .
$$

Mathematically, the reproducing field is described by the convolution of the pulse with a delta comb function. In the frequency domain this corresponds to the spectrum of

$$
\tilde{E}_{\text {train }}(f)=\tilde{A}\left(f-f_{c}\right) \cdot \sum_{m=-\infty}^{+\infty} \delta\left(f-m f_{\text {rep }}\right) \text {. }
$$

The spectrum of the pulse train for which an equidistant frequency comb is formed underneath the single pulse spectrum $\tilde{A}\left(f-f_{c}\right)$ is shown in Fig. 2 for $f_{\mathrm{CEO}}=0$. The comb lines are spaced by the pulse repetition frequency $f_{\text {rep }}$ and are given by integer multiples of $f_{\text {rep }}$, which mathematically corresponds to the delta comb function in (3). Note that a measurement of a single pulse spectrum would result in a continuous pulse spectrum $\tilde{A}\left(f-f_{c}\right)$ without the frequency comb.

Generally, $v_{g} \neq v_{p}$ because group and phase velocity inside the cavity both depend on the material and angular dispersion inside the cavity. When a pulse propagates through a dispersive medium with refractive index $n(x)$ and length $L$, its Fourier components experience a phase shift

$$
\psi(\omega)=-\int_{0}^{L} k(\omega, x) \mathrm{d} x=-\int_{0}^{L} \frac{n(\omega) \omega}{c} \mathrm{~d} x
$$

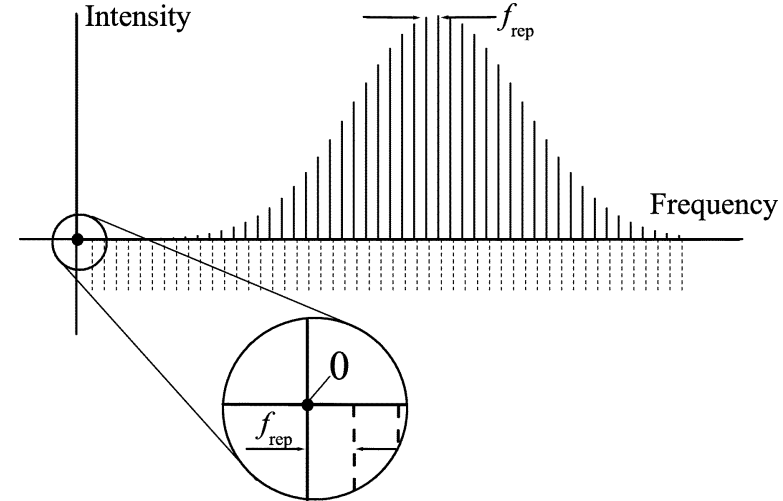

Fig. 2. Equidistant frequency comb of a mode-locked laser for equal group and phase velocities, i.e., the electric field pattern reproduces from pulse to pulse. Comb lines are are integer multiples of $f_{\text {rep }}$.

where $k$ is the wave number and $c$ the vacuum speed of light. From the fundamental definitions of phase velocity $v_{p}=\omega / k$ and group velocity $v_{g}=\mathrm{d} \omega / \mathrm{d} k$ one finds that

$$
\frac{1}{v_{g}}=\frac{1}{c}\left(\frac{\mathrm{d} n(\omega)}{\mathrm{d} \omega} \omega+n(\omega)\right)=\frac{1}{v_{p}}+\frac{\mathrm{d} n(\omega)}{\mathrm{d} \omega} \frac{\omega}{c} .
$$

Combining (4) and (5), we can calculate the phase lag between the pulse envelope and the carrier by integrating along the beam path of one cavity round trip

$$
\Delta \varphi_{\mathrm{GPO}}=-\omega \int_{0}^{\mathrm{L}}\left(\frac{1}{v_{g}}-\frac{1}{v_{p}}\right) \mathrm{d} x=\int_{0}^{L} \frac{\omega^{2}}{c} \frac{\mathrm{d} n(\omega, x)}{\mathrm{d} \omega} \mathrm{d} x .
$$

It is striking that this group-phase offset (GPO) only arises from the first-order dispersion of the material along the beam path. In a laser cavity, GPO contributions of all intracavity components add up. For a typical Ti:sapphire laser cavity the GPO contribution of the crystal alone is on the order of 1000 radians with additional components from mirror dispersion and the air path. Moreover, geometrical dispersion, i.e., a spectrally dependent path length, may add to the cavity round trip $\Delta \varphi_{\mathrm{GPO}}$ if elements such as prisms or gratings are used.

From (6) it follows that the group-phase offset phase $\varphi_{\mathrm{GPO}}$ changes by $\Delta \varphi_{\mathrm{GPO}}$ per round-trip time $T_{R}$, i.e.,

$$
\frac{\partial}{\partial t} \varphi_{\mathrm{GPO}}=\frac{\Delta \varphi_{\mathrm{GPO}}}{T_{R}} .
$$

As group-phase offsets of integer multiples of $2 \pi$ do not change the electric field pattern of a laser pulse, the quantity of interest is

$$
\Delta \varphi_{0}=\Delta \varphi_{\mathrm{GPO}} \bmod 2 \pi
$$

which describes the change of the carrier-envelope offset phase $\varphi_{0}$ in (1) per round trip. Even tiny variations of the intracavity material properties can give rise to variations of $\Delta \varphi_{0}$ due to the large value of $\Delta \varphi_{\mathrm{GPO}}$. The pulse-to-pulse fluctuations of $\Delta \varphi_{0}$ are extremely small as they correspond to changes during one round-trip time. However, for extended observation times these tiny variations may well add up to significant changes (see also the discussions concerning the observation time in Section VI). 


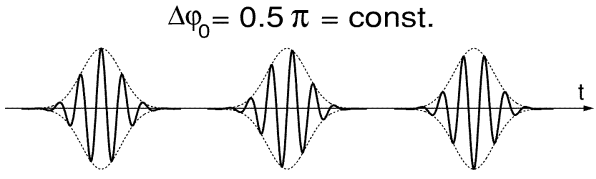

Fig. 3. Electric field $E(t)$ of three subsequent pulses from a mode-locked laser, i.e., the time-domain picture of the comb in Fig. 4. Envelope $\pm A(t)$ is shown as dashed lines. Here, the electric-field patterns of the pulses experience a constant pulse-to-pulse phase shift $\Delta \varphi_{0}=0.5 \pi$ while the value of $\varphi_{0}$ increases with time.

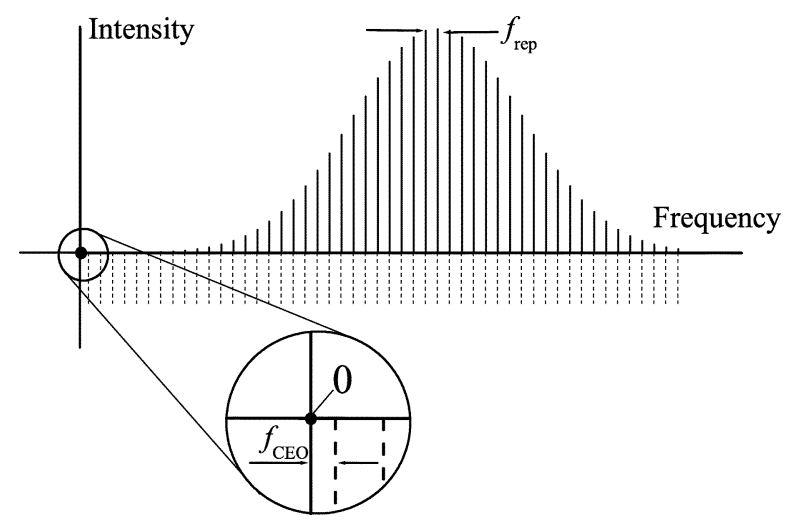

Fig. 4. Equidistant frequency comb of a mode-locked laser. Comb lines are spaced by the repetition rate $f_{\text {rep }}$ and exhibit a nonvanishing offset frequency $f_{\mathrm{CEO}}$ at zero frequency unless the electric field pattern exactly reproduces from pulse to pulse (compare to Fig. 3). Note the difference to the case of a vanishing CEO frequency as depicted in Fig. 2.

We define the (angular) carrier-envelope offset frequency as

$$
\begin{aligned}
2 \pi f_{\mathrm{CEO}} & =\omega_{\mathrm{CEO}}=\frac{\Delta \varphi_{0}}{T_{R}} \equiv \frac{\Delta \varphi_{\mathrm{GPO}}}{T_{R}} \bmod 2 \pi \\
& \equiv\left(\frac{\partial}{\partial t} \varphi_{\mathrm{GPO}}\right) \bmod 2 \pi
\end{aligned}
$$

Taking into account the varying carrier-envelope offset phase the electric field of the pulse train becomes (see Fig. 3)

$$
E_{\text {train }}(t)=A(t) \exp \left(i \omega_{c} t+i \omega_{\mathrm{CEO}} t\right) \otimes \sum_{m=-\infty}^{+\infty} \delta\left(t-m T_{R}\right)
$$

Using the Fourier shift theorem we can obtain the expression for the frequency domain

$$
\tilde{E}_{\text {train }}(f)=\tilde{A}\left(f-f_{c}\right) \cdot \sum_{m=-\infty}^{+\infty} \delta\left(f-m f_{\text {rep }}-f_{\mathrm{CEO}}\right) .
$$

The whole equidistant frequency comb of (3) is shifted by $f_{\mathrm{CEO}}$ due to the per round-trip carrier-envelope offset phase shift of $\Delta \varphi_{0}$. Fig. 4 depicts the spectrum of such a pulse train where we observe an equidistant frequency comb. All frequencies of the comb can be calculated from the simple equation

$$
f_{m}=m f_{\mathrm{rep}}+f_{\mathrm{CEO}} \text {. }
$$

The frequency comb spacing is equidistant and given by the round trip propagation time of the pulse envelope (i.e., by the group velocity and not the phase velocity). This means that the axial modes of a modelocked laser are not the same as the ones from a continuous wave $(\mathrm{cw})$ laser for which the phase velocity determines the axial mode spacing.

The uniformity of the mode-locked frequency comb has been demonstrated to a relative uncertainty below $10^{-15}$ [18]. Timing jitter in the arrival time of the pulses [19]-[22] produces a "breathing" of the otherwise fully equidistant frequency comb. On the other hand, the timing jitter of the $\mathrm{CEO}$ results in a translation of the full frequency comb.

\section{MEASUREMENT OF CEO FREQUENCY}

Even though a measurement of the repetition rate is straightforward, it is virtually impossible to access the CEO frequency directly, as the laser spectrum contains no energy close to zero frequency. One, therefore, has to use an indirect way to measure the second comb parameter via heterodyning of two pulse harmonics.

Let us examine a single pulse from the laser pulse train in the time domain. The electric field of the pulse can be described by (1) where $\varphi_{0}$ is the absolute phase of the pulse. This absolute phase must not be confused with the previously defined CEOphase, which was introduced as the absolute phase difference $\Delta \varphi_{\mathrm{CEO}}=\left.\varphi_{0}\right|_{t=T_{\mathrm{R}}}-\left.\varphi_{0}\right|_{t=0}$ of two subsequent pulses.

For a fixed phase shift for a single pulse at a fixed position in space, we can determine the pulse spectrum from (1)

$$
\tilde{E}(\omega)=\tilde{A}\left(\omega-\omega_{c}\right) \exp \left(i \varphi_{0}\right) .
$$

The field of the second-harmonic of $\tilde{E}(\omega)$ is then written as

$$
\begin{aligned}
\tilde{E}^{(\mathrm{SH})}(\omega) & =\gamma \int \tilde{E}\left(\omega^{\prime}\right) \tilde{E}\left(\omega-\omega^{\prime}\right) d \omega^{\prime} \\
& =\gamma \exp \left(2 i \varphi_{0}\right) \int \tilde{A}\left(\omega^{\prime}-\omega_{c}\right) \tilde{A}\left(\omega-\omega^{\prime}\right) d \omega^{\prime}
\end{aligned}
$$

where the proportionality factor $\gamma$ includes the second-order susceptibility and a constant phase shift. Note that the secondharmonic field $\tilde{E}^{(\mathrm{SH})}$ is centered at twice the fundamental carrier frequency $\omega_{c}$. The main conclusion is that absolute phases add up in a sum frequency generation and subtract in difference frequency generation. The latter has been exploited in optical rectification [23]. Difference frequency generation within one frequency comb cancels out the absolute phases of the generating pulses, which has also been demonstrated in mid-IR pulse generation [24], [25]. As the optical rectification process can be understood as difference frequency generation between spectral components from one and the same pulse, absolute phases subtract in optical rectification, which can be employed to synthesize terahertz or mid-IR pulses with a static absolute phase. Parametric amplification may also be used to generated pulses with static $\varphi_{0}$ [26].

Let us now consider a delayed superposition of both fields, $\tilde{E}^{(\mathrm{SH})}$ and $\tilde{E}$, at a frequency $\omega$ in the area of spectral overlap of the two harmonics

$$
\begin{aligned}
\tilde{I}(\omega) \propto & \left|\tilde{E}^{(\mathrm{SH})}(\omega)+\tilde{E}(\omega)\right|^{2} \\
= & \mid \exp \left(i \varphi_{0}\right) \tilde{A}\left(\omega-\omega_{c}\right) \\
& +\left.\gamma \exp \left(i \omega \Delta t+2 i \varphi_{0}\right) \tilde{A}^{(\mathrm{SH})}\left(\omega-\omega_{c}\right)\right|^{2} \\
= & \left|\tilde{A}\left(\omega-\omega_{c}\right)\right|^{2}+\gamma^{2}\left|\tilde{A}^{(\mathrm{SH})}\left(\omega-\omega_{c}\right)\right|^{2} \\
& +2 \gamma\left|\tilde{A}\left(\omega-\omega_{c}\right) \tilde{A}^{(\mathrm{SH})}\left(\omega-\omega_{c}\right)\right|^{2} \\
& \times \cos \left(\omega \Delta t+\varphi_{0}+\pi / 2\right) .
\end{aligned}
$$




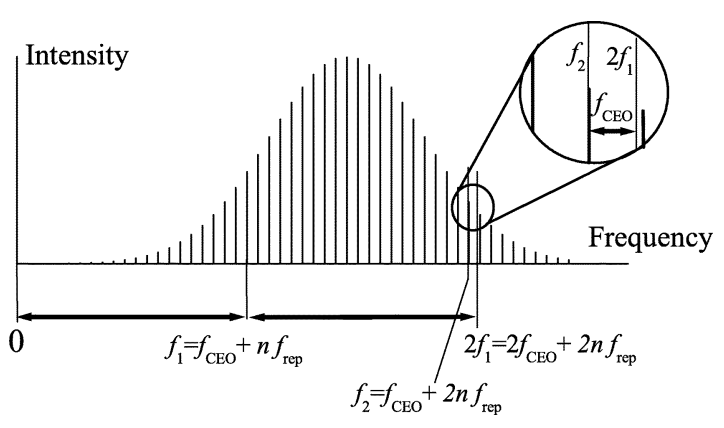

Fig. 5. Practical scheme for determining the CEO frequency of a laser comb. Scheme is based on (19), i.e., the case of heterodyning the fundamental and the second harmonic. Graphically, this scheme mirrors the origin at $f=f_{1}$, transferring the $f_{\mathrm{CEO}}$ beat from dc into a region with nonvanishing spectral content.

In the following, we will focus our interpretation on the cosine term in (15). This term gives rise to a spectral interference pattern if the delay $\Delta t$ is chosen a nonzero value. In principle, detection of this interference signal should allow for the determination of the absolute phase $\varphi_{0}$. Dispersive effects, however, will typically prevent a direct determination of the absolute phase using spectral interferometry. Still, this method allows monitoring shot-to-shot changes of $\varphi_{0}$, as has been described in [27] and [28]. Equation (15), therefore, holds the key for single-shot measurement of the CEO phase change $\Delta \varphi_{\mathrm{CEO}}$ in amplified laser systems.

Spectral interferometry cannot be directly used with typical laser oscillators because of the high repetion rates of oscillators. Therefore, we need to reconsider (15) and extend it to the multipulse case. Let us adjust $\Delta t=0$ and assume a linearly evolving absolute phase $\varphi_{0}(t)=2 \pi f_{\mathrm{CEO}} t$ (10) which corresponds to a constant shot-to-shot phase difference $\Delta \varphi_{\mathrm{CEO}}$. In this case, the interference term in (15) gives rise to a beat signal with constant frequency $f_{\mathrm{CEO}}$. This beat signal is exactly synchronous with relative phase changes between carrier and envelope of the pulse propagating through the laser cavity.

Note that none of the discussed methods allows determination of the absolute phase. In fact, all methods discussed so far only monitor changes of the absolute phase with time. Based on the previous discussion, the $f$-to- $2 f$ heterodyne scheme can be understood in a more figurative way if we consider heterodyning of two combs (Fig. 5) [7]. The laser comb consists of lines located at individual frequencies

$$
f_{m}=m f_{\text {rep }}+f_{\mathrm{CEO}} .
$$

Selecting some low-frequency portion of the comb and frequency doubling it gives rise to the comb

$$
2 f_{m}=2 f_{\mathrm{CEO}}+2 m f_{\mathrm{rep}} \text {. }
$$

If the fundamental comb spectrum covers more than an optical octave it will also contain modes at

$$
f_{2 m}=f_{\mathrm{CEO}}+2 m f_{\text {rep }} \text {. }
$$

Beating the combs of (17) and (18), therefore, extracts the CEO frequency from the comb spectrum

$$
2 f_{m}-f_{2 m}=f_{\mathrm{CEO}} .
$$

Note that this requires an octave-spanning spectrum, i.e., $f_{2 m} / f_{m}=2$.

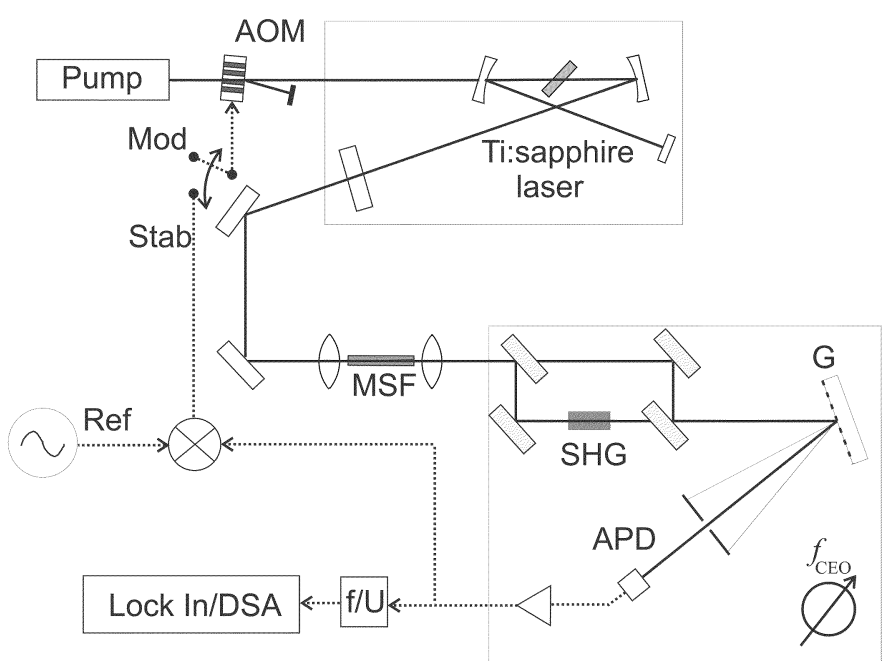

Fig. 6. Schematic setup used for tracking and stabilizing the CEO frequency of a femtosecond oscillator. AOM: acousto-optic modulator. MSF: micro-structure fiber. SHG: 1-cm long LBO crystal. G: grating. APD: avalanche photo diode. f/U: frequency-to-voltage converter. DSA: Fourier dynamic signal analyzer. Ref: quartz-stabilized rf synthesizer used to provide the reference frequency. Stab/Mod: settings used for stabilizing or modulating the CEO frequency, respectively.

The above procedure can be extended to the more general case of beating the $M$ th and the $N$ th harmonics of the comb spectrum. This yields $(M-N) f_{\mathrm{CEO}}$ as the beat signal and requires the comb spectrum to include a frequency ratio of $M / N$. Other more economic ways to deal with nonoctave-spanning spectra, such as transfer oscillators and interval bisection, are described in [7]. Another way of generating the necessary spectral width for application of the fundamental-SHG beating scheme is to use spectral broadening via self-phase modulation (SPM). This can be understood fully analogously to the previously discussed schemes of $\chi^{(2)}$ three-wave interaction. In SPM, three input waves of a frequency comb are mixed to yield a new component via four-wave interaction in a $\chi^{(3)}$ nonlinearity

$$
f_{m_{1}}+f_{m_{2}}-f_{m_{3}}=f_{\mathrm{CEO}}+\left(m_{1}+m_{2}-m_{3}\right) f_{\mathrm{rep}} .
$$

It is fairly obvious that this process can generate new frequency components while still maintaining the original CEO frequency of the comb. Note that the same argumentation holds for the case of low-repetition rate sources, which are CEO-tracked by spectral interferometry [27], [28].

\section{IV. $f$-TO- $2 f$ CEO HETERODYNING TECHNIQUE}

The general setup of a CEO tracking scheme is depicted in Fig. 6. In nearly all cases, the laser source is initially broadened employing either microstructured fibers [29] or hollow gas-filled fibers [30]. Subsequently, one generates two different harmonics from the spectrally broadened beam. For simplicity, the fundamental and the second harmonic are most frequently used. The two harmonics are then heterodyned in an interferometer as shown in Figs. 5 and 8. It depends on the application whether to slightly unbalance the arm lengths in the Michelson interferometer and then detect the entire spectrum [27] or to match the arm lengths and detect the interferometer output with a single photo detector [7]. 


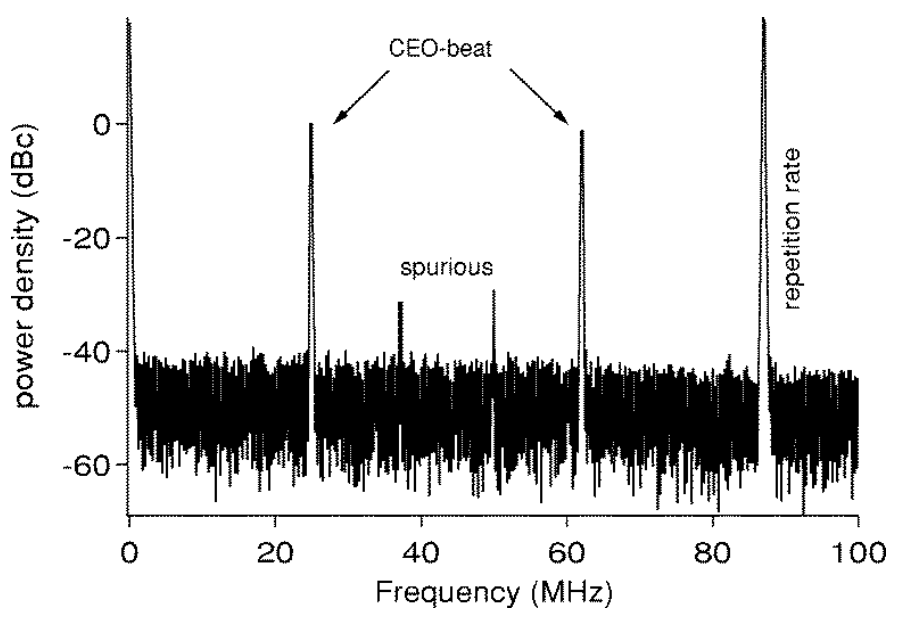

Fig. 7. Typical rf spectrum of the heterodyne signal between fundamental and second harmonic. Spectrum contains some spurious contributions from mixing processes in the detector electronics. CEO frequency and its mirror are clearly visible at more than $40 \mathrm{~dB}$ above noise level at 25 and $62 \mathrm{MHz}$, respectively.

The latter approach is used to measure the CEO frequency of a laser oscillator, and it is only useful for laser repetition rates in the megahertz range. The former approach of spectral interferometry can track CEO-phase changes from pulse to pulse but requires detection of single-shot spectra. The spectral interference pattern needs to be static for the exposure time of the spectrometer array, as otherwise the interference contrast will vanish. This restricts the use of the former method to repetition rates in the kilohertz range and below.

Fig. 7 shows a typical rf spectrum measured by the cw heterodyning setup. The CEO beat note and its mirror frequency are clearly visible at 25 and $62 \mathrm{MHz}$. The measured signal level is $40 \mathrm{~dB}$ above the noise floor in a $100-\mathrm{kHz}$ bandwidth. In our experiments, we found a signal level of about $30 \mathrm{~dB}$ in a $100-\mathrm{kHz}$ measurement bandwidth necessary for reliable locking of the carrier-envelope offset frequency. Note that a smaller measurement bandwidth reduces the noise level with respect to the CEO beat signal but does not improve the stabilization because the CEO frequency stabilization needs a large bandwidth (see Section VI). In some early experiments, we observed that this beat frequency may change very rapidly by up to several megahertz in $1 \mathrm{~s}$. This behavior is already strongly reduced by enclosing the laser in a box. Excursions of the CEO frequency are further decreased in a prismless laser setup. With these improvements and still without any active stabilization, the CEO frequency stays within a $500-\mathrm{kHz}$ interval for observation times of several minutes. The major contribution to residual fluctuations are slow drifts of environmental parameters like air pressure, crystal temperature, etc. (Fig. 8).

\section{Physical Origin of CEO FreQuency Fluctuations}

As will become clear in the following experimental sections, the CEO frequency is an extremely sensitive parameter, which will strongly respond to the slightest environmental changes of the laser cavity. The CEO frequency can display excessive fluctuations in unstabilized femtosecond oscillators. Control of the CEO phase with a rigid lock to an rf reference is, therefore, very important not only in frequency metrology but also for nonlinear

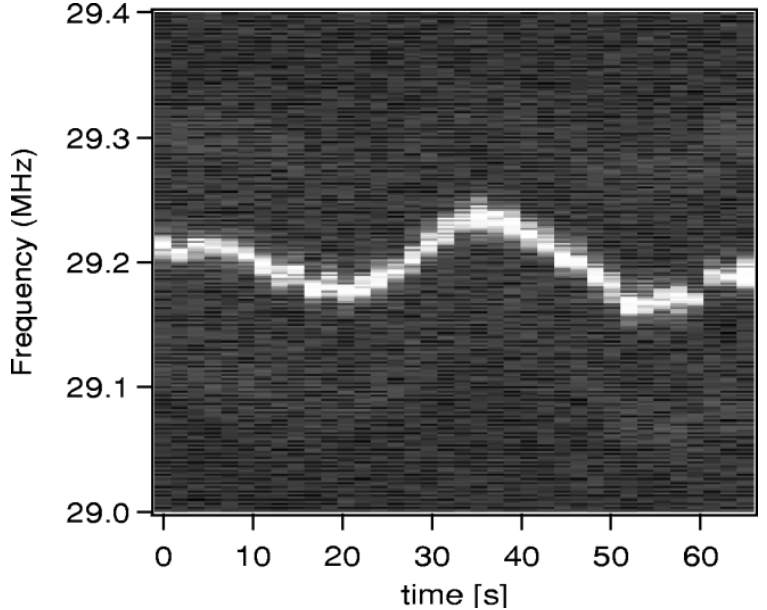

Fig. 8. Typical passive stability of the CEO frequency.

optics applications as already discussed in the introduction. For better stabilization it helps to understand the physical origin of these CEO fluctuations [8], [9], which are mainly caused by variations of intracavity dispersion. In this section, we will investigate how changes of dispersion affect the CEO frequency.

Any change of the linear intracavity dispersion will affect the $\Delta \varphi_{\mathrm{GPO}}$ via (6) and give rise to a variation of the CEO frequency. Changes of the cavity length, e.g., may also affect the $\mathrm{CEO}$ frequency via its dependence on the repetition rate. However, this effect is typically orders of magnitude weaker than contribution from dispersion. It should be noted that the connection between dispersion and the CEO frequency gives rise to coupling of laser power noise to CEO fluctuations via nonlinear optical effects. However, one can also employ the very same coupling to counteract fluctuations of the CEO frequency and to achieve active control of this laser parameter. A more detailed discussion is given in our previous publications [8], [9].

\section{CEO NOISE MEASUREMENTS}

To quantify the strength of CEO fluctuations in a Ti:sapphire laser, we set up the heterodyne detection scheme described in detail before. In most of the experiments, we use a Ti:sapphire oscillator similar to the setup described in [2]. One variant of the laser uses a sequence of two fused silica prisms in combination with chirped mirrors for dispersion compensation. Alternatively, we use the same laser converted to a prismless scheme, in which only chirped mirrors are employed for dispersion compensation. Both lasers deliver pulses in the 10-20-fs duration regime with a few $100 \mathrm{~mW}$ of output power. For the calculation of amplitude-to-phase coupling effects the peak intensity inside the 2.3-mm-long Ti:sapphire gain crystal is the decisive parameter. This value was calculated from the spatial and temporal pulse parameters as $4.5 \times 10^{11} \mathrm{~W} / \mathrm{cm}^{2}$ for the laser with prisms and $10^{12} \mathrm{~W} / \mathrm{cm}^{2}$ for the prismless laser. Except for the mirrors used in the laser, the pump geometry and the mechanical setup have been left widely unchanged to allow for a comparison of the two lasers.

We electronically convert the CEO frequency into a voltage, employing a phase-locked loop. This voltage is then spectrally analyzed by a dynamic signal analyzer (HP3562A). Multiplying the measured voltage noise by the conversion factor of the 


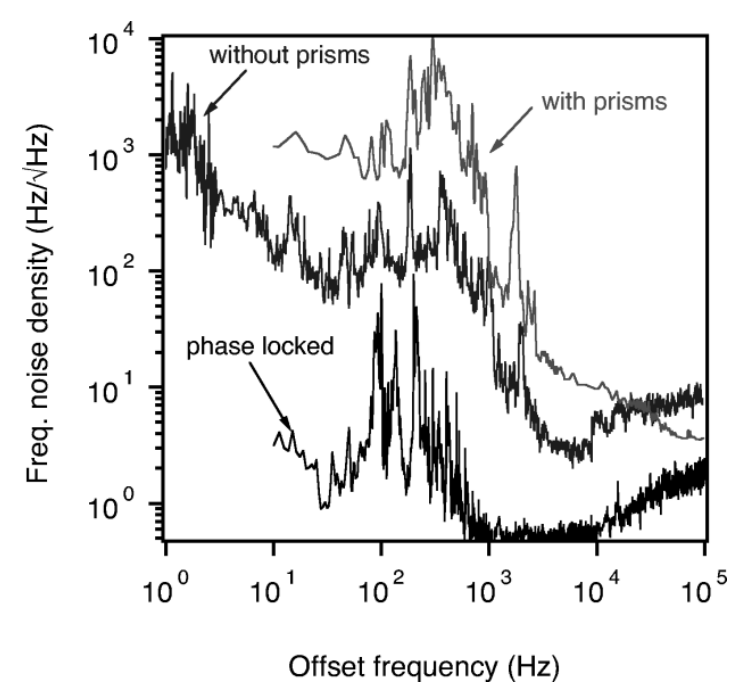

Fig. 9. CEO frequency noise density versus Fourier frequency of different femtosecond lasers. Top trace depicts the noise of an unstabilized oscillator with intracavity prisms for dispersion compensation. Bottom and the middle trace shows the noise of a laser with mirror-only dispersion compensation, with and without stabilization, respectively. All traces were determined with a frequency-to-voltage converter.

frequency-to-voltage converter yields the single-sideband frequency noise density $\sigma_{f \mathrm{CEO}}(f)$ in units $\mathrm{Hz} / \sqrt{\mathrm{Hz}}$ versus offset frequency $f$. Fig. 9 shows measurements of the CEO frequency noise of the free-running laser with intracavity prisms and the prismless laser with and without stabilization. All measurements are composed of several sweeps with different spectral resolutions and are combined in a logarithmic plot, covering the range from $1 \mathrm{~Hz}$ to $100 \mathrm{kHz}$. The noise spectra typically show some discrete components at line frequency harmonics and a broad background reaching up to several kilohertz offset frequency. The laser with intracavity prisms shows by far the worst noise behavior with a pronounced maximum centered at about $500 \mathrm{~Hz}$. The prismless laser shows a more than ten times improved passive stability. An explanation of this dramatic reduction is given by additional contributions from beam pointing variations, which can translate into CEO phase noise if intracavity geometrical dispersion compensation elements are present [8], [9]. As prism or grating sequences employ wavelength-dependent beam deflection to generate a negative dispersion, beam pointing variations will couple into changes of the dispersion, including first-order dispersion. This effect is avoided in prismless cavities, which only use chirped mirrors for dispersion compensation.

A further reduction of the noise can be achieved with active stabilization, which is shown for comparison and will be discussed below.

Let us assume that the CEO frequency has been monitored for a time period $T_{\text {meas }}$, resulting in a value $\bar{f}_{\mathrm{CEO}}=\left(\varphi_{0}\left(T_{\text {meas }}\right)-\right.$ $\left.\varphi_{0}(0)\right) /\left(2 \pi T_{\text {meas }}\right)$. If we compare the $N=T_{\text {meas }} / T_{R}$ measurements of the instantaneous CEO phase $\varphi_{0}(t)$ with a linearly evolving phase $\varphi_{0}(0)+2 \pi \bar{f}_{\mathrm{CEO}} t$, jitter of the laser may lead to an instantaneous phase deviation

$$
\varepsilon\left(t=q T_{R}\right)=2 \pi \bar{f}_{\mathrm{CEO}} t-\sum_{l=0}^{q-1} \Delta \varphi_{\mathrm{CEO}}\left(l T_{R}\right) .
$$

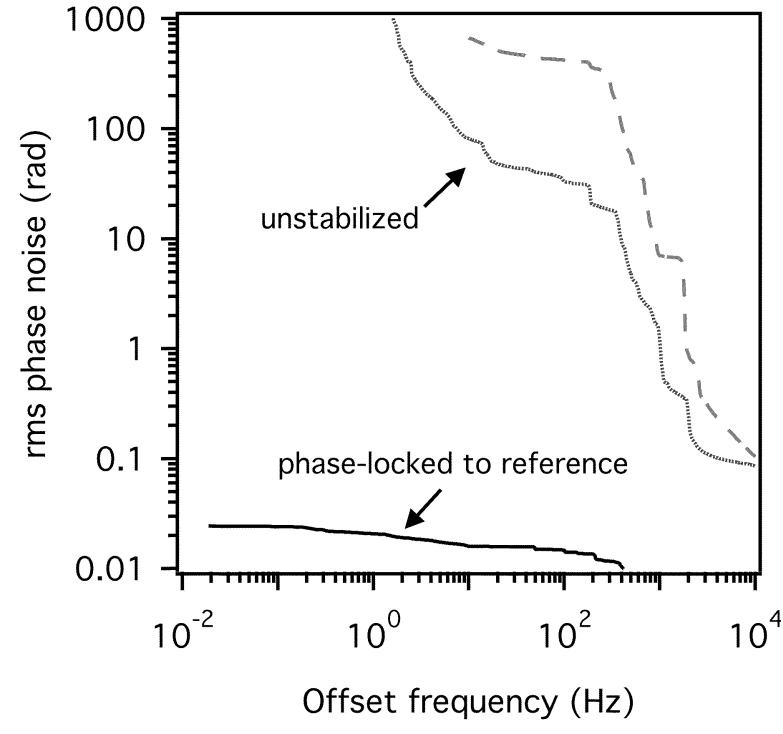

Fig. 10. Root mean square phase noise for the three cases shown in Fig. 9. Top trace (unstabilized laser with prisms) and middle trace (unstabilized prismless laser) have been directly integrated from the measurements in Fig. 9 according to (23). Bottom trace for the prismless stabilized laser is independently measured using homodyne detection employing an rf lockin.

From measurements of $\varepsilon$, one can derive an estimation of the phase fluctuations for any given measurement period $T_{\text {meas }}$

$$
\delta_{\varphi_{\mathrm{CEO}}}\left(T_{\text {meas }}\right)=\sqrt{\frac{2}{N} \sum_{l=0}^{N-1} \varepsilon^{2}\left(l T_{R}\right) .}
$$

The quantity $\delta_{\varphi_{\mathrm{CEO}}}\left(T_{\text {meas }}\right)$ can also be obtained by analyzing the fluctuations in the frequency domain, i.e., measuring the amount $\sigma_{\varphi_{\mathrm{CEO}}}(f)$ which characerizes how much $\varphi_{0}(t)$ fluctuates at a given Fourier frequency $f$ during the observation period $T_{\text {meas }}$

$$
\delta_{\varphi \mathrm{CEO}}\left(T_{\text {meas }}\right)=\sqrt{2 \int_{f_{\text {low }}}^{f_{\text {high }}} \sigma_{\varphi_{\mathrm{CEO}}}^{2}(f) \mathrm{d} f}
$$

with $f_{\text {low }}=1 / T_{\text {meas }}$ being the lowest and $f_{\text {high }}=f_{\text {rep }}=$ $1 / T_{R}$ the highest possible Fourier frequency during the observation time $T_{R}$. The phase noise density can be computed from the measured frequency noise density as ${ }^{1}$

$$
\sigma_{\varphi_{\mathrm{CEO}}}(f)=\frac{\sigma_{f \mathrm{CEO}}(f)}{f} \text {. }
$$

Data for the integrated phase noise determined from Fig. 9 by using (23) is shown in Fig. 10. The integration is always carried from a variable lower bound $f_{\text {low }}$ to an upper bound $f_{\text {high }}$. The upper bound $f_{\text {high }}$ is determined by the detection bandwidth and can be as high as the pulse repetition frequency $f_{\text {rep }}$. The lower bound $f_{\text {low }}$ is determined by the measurement duration or integration time as discussed above. The longer the measurement takes, the lower $f_{\text {low }}$ is. Given the rapid noise rolloff toward high frequencies, the bandwidth of our analyzer does not appear to obscure significant contributions to the rms phase jitter. The data in Fig. 10 renders a graphical display for the severity

\footnotetext{
${ }^{1}$ Conversion from frequency noise densities to phase noise densities contained an erroneous factor $2 \pi$ in [9, eq. (6)]. This factor is now removed. Experimental data in Fig. 10 is now scaled accordingly.
} 
of the CEO phase noise issues. In the unstabilized lasers, the CEO phase noise exhibits a divergence toward zero frequency, roughly following a $1 / f$ dependence. A $2 \pi$ cumulated phase noise is already reached at observation times below $1 \mathrm{~ms}$. If the linear trend of the phase versus time is known from a CEO frequency measurement, the jitter in an unstabilized laser will totally wash out the CEO phase within a fraction of $1 \mathrm{~ms}$. Any experiment that will require a constant or controlled CEO phase for longer than $1 \mathrm{~ms}$ will require an active stabilization of this quantity.

We can also conclude from Fig. 10 that CEO phase fluctuations have rather high-frequency components compared to timing jitter of the pulse repetition period [19]-[22]. Typically solid-state lasers only exhibit significant timing jitter of the arrival time of the pulses below $1 \mathrm{kHz}$ [20], [31]. Therefore, we will require a minimum control bandwidth of several kilohertz to establish an active servo lock to stabilize the timing jitter of the CEO phase. This clearly favors acousto-optic or electrooptic mechanisms for setting the CEO frequency.

\section{Controlling the CEO FReQuency of A FEMTOSECOND LASER}

The question arises as to what mechanisms can be used to counteract fluctuations of the CEO frequency. Such a means of control is the prerequisite for any type of active stabilization. The problem in CEO stabilization is less a problem of finding an effect to control the CEO frequency, but more one of selectively and rapidly changing only this laser pulse parameter.

Tilting of the end mirror of the prism arm of the laser was described as one of the first methods to adjust the CEO frequency [32]. This method has the advantage of minimum impact on other laser pulse parameters, but it is limited by the inertia of the mirror. Mirror tilting is actually an example for group-phase offset contributions from geometrical dispersion [33]. Unfortunately, this method cannot be used in a prismless laser setup.

The CEO frequency can also be changed by an adjustment of the laser pump power as was first proposed in [13]. Originally, the change of CEO frequency with pump power was explained by a common-mode shift of the laser spectrum. Together with the intracavity dispersion, this type of laser dynamics would give also rise to a changing CEO-phase. Nevertheless, such a spectral shift is not observed in all mode-locked lasers. A similar effect also arises from group-phase contributions from the Kerr-effect. Here, the varying peak power of the intracavity pulses affects the dispersive properties of the gain medium [8], [9].

Fig. 11 shows a transfer function, i.e., the measured change of CEO frequency induced by an acousto-optic amplitude modulator, which is located between pump laser and the Ti:sapphire laser. The transfer function displays a reduction of the coupling coefficient from about $10^{-7} \mathrm{~Hz} \mathrm{~m}^{2} / \mathrm{W}$ to $10^{-8} \mathrm{~Hz} \mathrm{~m}^{2} / \mathrm{W}$. We attribute coupling of the intracavity peak power and CEO phase mainly to intensity-induced changes of the refractive index. At low frequencies, contributions from a thermally induced index change may be present, which disappear at higher modulation frequencies. In the latter case, only contributions from the electronic contribution to the refractive index, i.e., the Kerr effect,

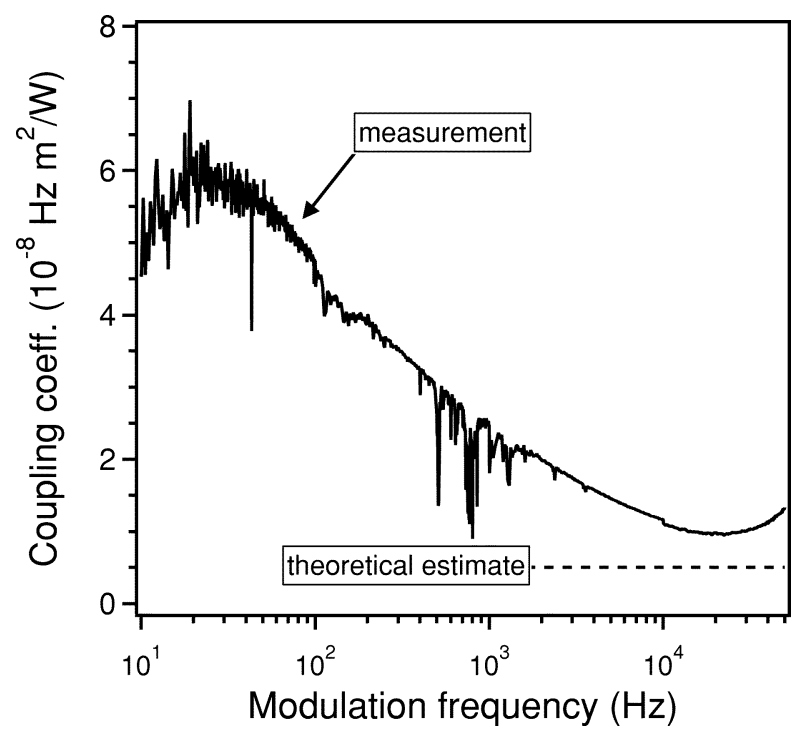

Fig. 11. Transfer function from intracavity intensity modulation to CEO frequency change for the range from $10 \mathrm{~Hz}$ to $50 \mathrm{kHz}$. Modulation is induced by an acousto-optic modulator between pump laser and a Ti:sapphire oscillator. Pump laser modulation is converted to units of the peak intensity change inside the laser crystal. CEO frequency was monitored employing the scheme described in Section IV.

will appear. It is well known [34] that a temperature change affects the group index and the phase index of a material in a different way. This causes a thermally dependent group-phase offset and affects the measured CEO frequency according to (9).

Normally, the Kerr effect is treated in a nondispersive way [35], which totally suffices to describe isolated effects of either the envelope or the phase. Nevertheless, it has to be anticipated that intensity-induced as well as temperature-induced electronic group and phase changes are not identical. This means that the Kerr effect also exhibits an intensity-dependent group-phase offset. From the previous discussions it becomes clear that we have to deal with the difference between group and phase delays or the linear dispersion of the Kerr effect. The dependence of the nonlinear refractive $n_{2}$ index versus wavelength has been subject of both experimental [36] and theoretical investigations [37].

Based on the theoretical model for the spectral dependence $n_{2}(\omega)$ in [37], one can define a nonlinear contribution to the GPO as

$$
\Delta \varphi_{\mathrm{GPO}}^{(\mathrm{nl})}=\frac{L \omega^{2}}{c} I \frac{\mathrm{d} n_{2}(\omega)}{\mathrm{d} \omega}
$$

where $I$ is the intensity in the Kerr medium. This contribution has to be put in relation with the SPM-induced phase shift

$$
\Delta \varphi_{\mathrm{SPM}}=\frac{L \omega}{c} n_{2} I=\Delta \varphi_{\mathrm{GPO}}^{(\mathrm{nl})} \frac{n_{2}}{\omega \mathrm{d} n_{2} / \mathrm{d} \omega} .
$$

For sapphire at $800-\mathrm{nm}$ center wavelength, one calculates $\omega \mathrm{d} n_{2} / \mathrm{d} \omega=8 \times 10^{-17} \mathrm{~cm}^{2} / \mathrm{W}$. This means that the contribution of the Kerr nonlinearity to the group-phase offset is about five times smaller than SPM assuming $n_{2}=3.6 \times 10^{-16} \mathrm{~cm}^{2} / \mathrm{W}$ [35], [37]. Plugging in numbers for the experimental conditions of Fig. 11, one further estimates $\mathrm{d} f_{\mathrm{CEO}} / \mathrm{d} I=4 \times 10^{-5}$ $\mathrm{Hz}$ per $\mathrm{W} / \mathrm{cm}^{2}$. This agrees well with the measured value $\mathrm{d} f_{\mathrm{CEO}} / \mathrm{d} I \approx 10^{-4} \mathrm{~Hz} \mathrm{~cm}^{2} / \mathrm{W}$ in Fig. 11 . 


\section{CEO PHASE LOCK}

The schematic setup of the servo loop in our experiments is depicted in Fig. 6. The measured CEO beat signal is first electronically cleaned by suitable band-pass filters and then amplified. The processed signal is mixed with a reference oscillator using a double-balanced mixer (MiniCircuits ZP3). The loop is closed by feeding the intermediate-frequency signal into the input of the acousto-optic pump power modulator. It is important to adjust the overall loop gain for a sufficient phase margin. This suppresses the parasitic oscillation of the stabilization scheme.

In the case of active stabilization of the CEO frequency, a direct measurement of the phase noise density with an rf lock-in is generally far superior to the frequency-to-voltage conversion scheme described before. As a phase reference is available, a homodyne measurement provides much more reliable information at low offset frequencies. These homodyne noise measurements can be easily extended into the megahertz range. We directly measured the phase noise jitter $\delta \varphi_{\mathrm{CEO}}$ with a Stanford SR844 rf lock-in (phase sample frequency $20 \mathrm{kHz}$ ). This data is also shown in Fig. 10 and can be easily compared with the measurements of the free-running laser. Our measurements indicate that the measured CEO phase of the stabilized oscillator is always kept within an rms range of 25 mrad relative to the reference oscillator, up to integration times of one minute. Translating this phase jitter back to a timing jitter, one finds that a 25 -mrad jitter corresponds to a relative timing jitter between carrier and envelope

$$
\delta t_{\mathrm{CEO}}=\frac{\delta \varphi_{\mathrm{CEO}}}{\omega_{c}}
$$

of less than 10 attoseconds. It has to be emphasized that such a rigid lock can only be achieved with a double-balanced mixer, but not by phase locks with extended phase locking range, as have been used by other groups [16]. The tradeoff is that the more rigid lock also requires a higher passive stability of the laser.

While a residual jitter of 10 attoseconds is clearly the lowest value reported in literature for extended observation times, one has to be well aware of the limitations of the measurement scheme employed. First, it has to be noted that in the above measurements, the noise diagnostics and the stabilization circuitry were completely separated to allow for an independent check of the quality of the stabilization. However, the optical part of the measurement setup was not duplicated in these experiments. In particular, air turbulence in the Michelson interferometer may cause spurious drift components to the measured signal. We expect this not to be a severe effect, and it can be easily avoided by switching to a common path interferometer as used in single shot CEO measurements.

Another spurious contribution arises because of amplitude-to-phase coupling effects inside the microstucture fiber (MSF). These effects originate from the strong nonlinearity of the continuum generation process. Even tiny variations of the pulses entering the MSF can cause major changes of the spectral phase of the supercontinuum [38] and thus alter the measured CEO signal even though the CEO frequency of the

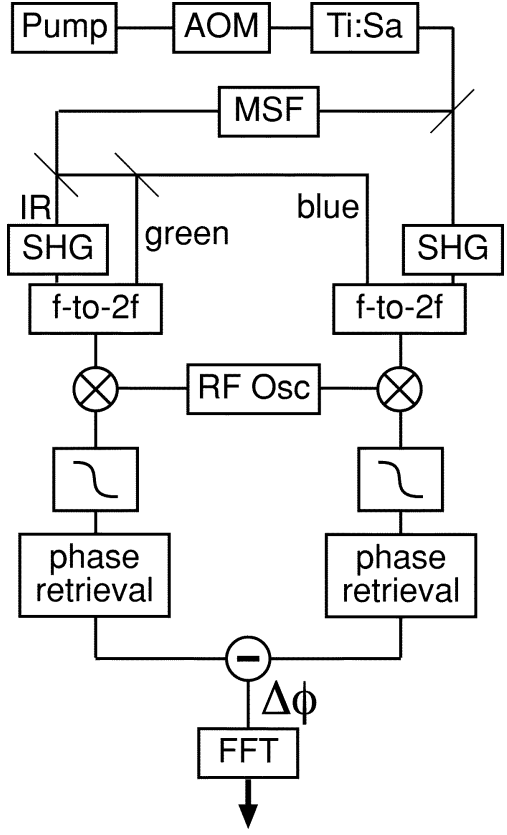

Fig. 12. Setup schematic. AOM: acousto optic modulator, Ti:Sa: Ti:Sa oscillator, MSF: microstructure fiber, SHG: second harmonic generation, f-to-2f: interferometer measuring the CEO frequency, RF Osc: RF synthesizer.

oscillator was constant. Thus, strictly speaking, only the pulses exiting the microstructure fiber are CEO stabilized, but not those entering the fiber, which exhibit an additional CEO phase noise contribution.

For a measurement of the strength of contributions from amplitude-to-phase conversion in the fiber, we track the phase difference between two differently implemented CEO frequency measurement schemes (see Fig. 12). One of these schemes generates the second harmonic from the light that propagated through the fiber, the other scheme generates the second harmonic directly from the oscillator output. The latter second harmonic, therefore, has not experienced any amplitude-to-phase coupling artifacts in the microstructure fiber. Both second harmonics are heterodyned with corresponding fundamental portions of the fiber continuum, as described in Section III. To ensure full independence of both measurement setups and for maximum signal-to-noise ratio, we used different wavelengths for the two independent heterodyning schemes. In the following, the scheme that employs SHG from the laser oscillator will be referred to as the "blue" scheme, the other one the "green" scheme.

In the experiments, a 4-cm piece of a microstructure fiber manufactured by Crystal Fibers is employed for continuum generation. The CEO frequency of the free-running oscillator is coarsely adjusted to $30 \mathrm{MHz}$ by tilting an Brewster-angled intracavity glass plate, which is also used for fine tuning of intracavity dispersion. Both RF CEO frequencies, green and blue, are mixed with the same 29.8-MHz local oscillator using double balanced mixers (MiniCircuits ZP3) and low-pass filtered, resulting in downshifted frequencies at about $200 \mathrm{kHz}$. Due to the symmetric mixing scheme employing the same local oscillator, phase differences between the "green" and "blue" CEO frequencies are conserved in the downshifted IF signals. Both 


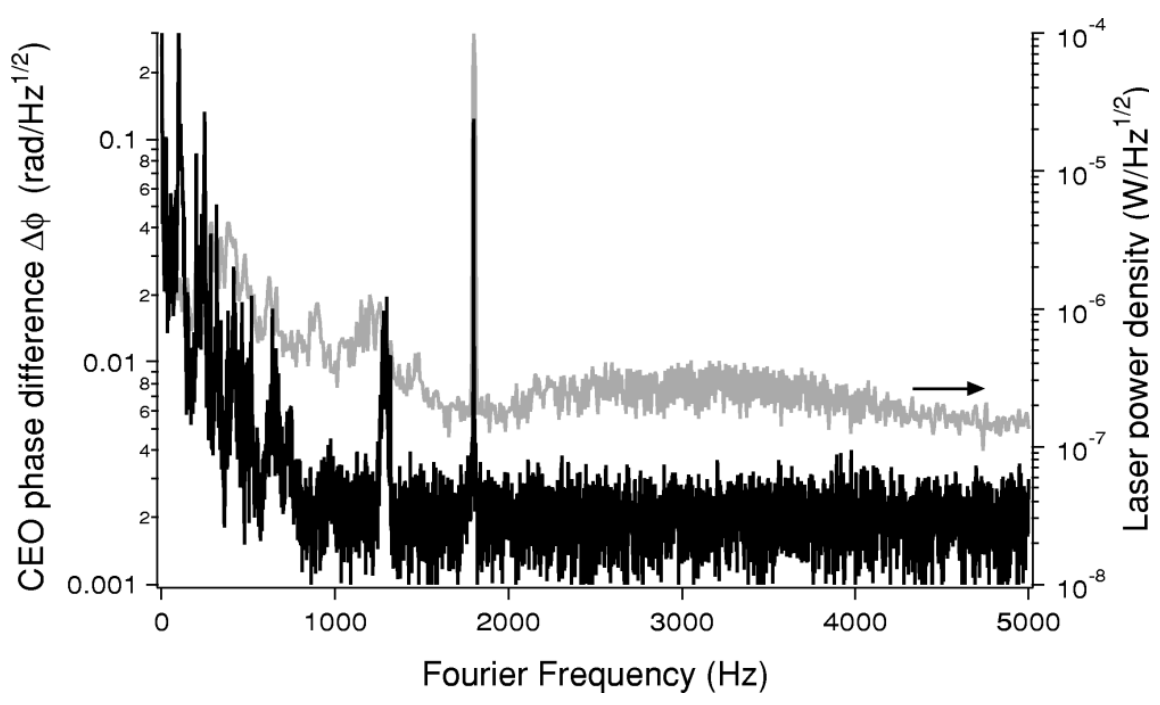

Fig. 13. Spectra of the phase difference $\Delta \phi$ between the two measured CEO frequencies and the laser power.

signals are then simultaneously digitized with a 12-bit resolution at a $6.7 \mathrm{MSa} / \mathrm{s}$ sampling rate. Finally, the phases of both 200-kHz IF signals are retrieved by a Fourier filtering technique [39]. Fig. 13 shows a typical example of such a measurement on the free-running laser. The two different detection schemes accumulate a phase difference of a few radians in an observation time of $2.5 \mathrm{~s}$.

It is important to note that this phase drift is a relatively weak effect, compared to the phase fluctuations of the free-running oscillator itself, which easily accumulates 1000 times more phase jitter in the same time interval. Nevertheless, the drift effect in the detection is significant compared to the residual jitters observed in the stabilization experiments. Generally, a phase difference between two independent CEO measurement schemes could also stem from a slight relative phase drift $\Delta \phi$ of the two separate interferometers. To isolate nonlinear drift contributions from fiber nonlinearities, we modulate the oscillator power at a perturbative level of less than $0.1 \%$. The $\Delta \phi$ signal then shows oscillations at the modulation frequency, which is a clear proof of peak power-dependent contributions from nonlinear amplitude-to-phase-coupling processes to the measured CEO frequency signals. Fig. 13 shows spectra of $\Delta \phi$ and the laser power. Apart from the sharp needle at the modulation frequency of $1.8 \mathrm{kHz}$, the noise floor of both spectra exhibits a strong similarity. This similarity further supports the importance of amplitude-to-phase conversion in the microstructure fiber. Evaluating a series of measurements, we compute coupling coefficients $\Delta \phi$ per oscillator peak power change ranging from 1 to $1.6 \mathrm{rad} / \mathrm{mW}$ with an average value of $1.17 \mathrm{rad} / \mathrm{mW}$. The $\mathrm{rms}$ power noise of the noise floor of spectrum depicted in Fig. 13 (without the peak at $1.8 \mathrm{kHz}$ ) is evaluated as $0.17 \mathrm{~mW}(6 \mathrm{~Hz}-5 \mathrm{kHz})$. With the coupling constant of $1.17 \mathrm{rad} / \mathrm{mW}$, even such a small jitter already translates to 0.2-rad rms phase noise between the two CEO measurement paths, introduced solely by amplitude-to-phase conversion of intrinsic laser noise.

As a consequence, one should always try to use the continuum directly for an experiment to avoid phase jitter in the fiber itself, be it in metrology or in extreme nonlinear optics. This

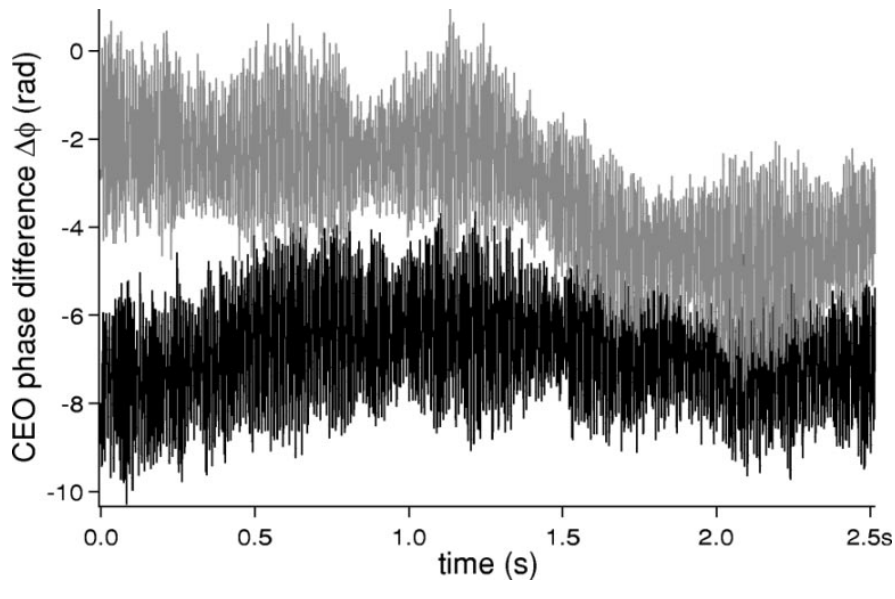

Fig. 14. Two traces of the phase difference $\Delta \phi$ between the "green" and "blue" CEO frequency without pump power modulation. Phase drifts exhibits a slow drift which is very dependent on the environmental circumstances but on the order of $1 \mathrm{rad} / \mathrm{s}$. Additional oscillation with an amplitude of about $1 \mathrm{rad}$ is present due to amplitude-to-phase coupling from pronounced low-frequency laser power noise (compare to Fig. 13).

further encourages use of sources that generate the necessary bandwidth directly, which, however, exhibit other drawbacks: a $f$-to- $2 f$ scheme demonstrated in [16] performed poorly in terms of phase jitter. The technically more demanding $2 f$-to- $3 f$ scheme used in [17] exhibited an improved phase stabilization, but the low pulse energy due to the $1-\mathrm{GHz}$ repetition rate of the used oscillator is disadvantageous for experiments in the extreme nonlinear optics regime.

If the laser output is used directly, but a continuum generation is used in the CEO-detection scheme, the resulting error budget in our experiments is on the order of $1 \mathrm{rad} / \mathrm{s}$ (compare to Fig. 14) with its exact value being strongly dependent on various environmental and laser parameters during the measurement. This value would limit metrological applications to $10^{-15}$ precision if the frequency measurement is not done by beating the fiber continuum with the frequency to be measured. Experiments in extreme nonlinear optics would be restricted to integration times of about $1 \mathrm{~s}$. Even though the CEO stabilization of oscillators 
was reported to reduce power noise above Fourier frequencies of a few kilohertz [8], [40], the large residual power noise at low Fourier frequencies will render the CEO phase of the oscillator pulses fluctuating, regardless of the quality of the CEO stabilization after the microstructure fiber.

In contrast to the measurement scheme demonstrated before [41], [42], our scheme did not require a stabilization of the CEO frequency and thus minimized laser perturbations as it does not need any servo loop. Moreover, using only one microstructure fiber avoids measurement errors resulting from differences of the coupling into the fibers as well as from their different properties. In this sense, this data is an improved analysis of amplitude-to-phase coupling effects in continuum generation, the process that is at the very core of nearly every demonstrated CEO measurement reported so far.

\section{Ultrashort Pulses and Frequency Metrology}

The relative phase between the carrier and the envelope of an optical pulse is the key parameter linking the fields of precision frequency metrology and ultrafast laser physics. As we have discussed in Section II, the spectrum of a mode-locked laser consists of a comb of precisely equally spaced frequencies. The uniformity of this frequency comb has been demonstrated to a relative uncertainty below $10^{-15}$ [18]. Knowledge of only two parameters, the comb spacing and a common offset frequency of all modes, provides one with a set of reference frequencies, similar to the tick marks on a ruler. Any unknown optical frequency within the comb bandwidth is easily compared with the neighboring comb frequencies by heterodyning source and reference on one and the same detector. This greatly simplifies the measurement of an optical frequency, which now requires the measurement of only three radio-frequency difference frequencies. The mode-locked laser provides a phase-locked clockwork mediating between radio frequencies and the Terahertz frequencies of the lines in the optical comb, effectively rendering optical frequencies countable. Details on precision frequency measurements with mode-locked laser combs can be found in [32] and [43]-[46].

\section{CONCLUSION}

We have investigated fluctuations of the CEO phase in oscillators and explained some of the major driving forces behind this type of phase noise. From the observations reported so far, four main conclusions have to be drawn.

1) Intracavity prism sequences additionally contribute to the CEO phase noise by a contribution from beam pointing noise. Lasers employing prism sequences for dispersion compensation therefore exhibit much higher CEO noise levels than prismless oscillators.

2) Amplitude fluctuations are coupled into CEO phase variations by various mechanisms, including thermal contributions and nonlinear refraction.

3) The contributions from nonlinear refraction agree well with the dispersion of the Kerr effect based on the theoretical model of [37].

4) Amplitude-to-phase coupling effects inside the microstructure fiber have to be taken into account when employing oscillator pulses for absolute phase dependent nonlinear experiments or precision metrology.

Based on our analysis, we achieved a phase lock of the CEO frequency to an external $\mathrm{rf}$ reference oscillator. The residual phase jitter between laser and reference was found to be less than $20 \mathrm{mrad}$, for integration times up to $1 \mathrm{~min}$. This small jitter corresponds to a timing jitter between carrier and envelope of approximately 10 attoseconds. The demonstrated control of the CEO phase with attosecond residual timing jitters is an important prerequisite for experiments in extreme nonlinear optics and precision frequency metrology.

\section{ACKNOWLEDGMENT}

The authors would like to thank R. S. Windeler, Lucent Technologies, and Ove Poulson, NKT Research, for microstructure fibers. They also gratefully acknowledge support by H. R. Telle, J. Stenger, and B. Lipphardt from the PTB frequency metrology group.

\section{REFERENCES}

[1] G. Steinmeyer, D. H. Sutter, L. Gallmann, N. Matuschek, and U. Keller, "Frontiers in ultrashort pulse generation: Pushing the limits in linear and nonlinear optics," Science, vol. 286, pp. 1507-1512, 1999.

[2] D. H. Sutter, G. Steinmeyer, L. Gallmann, N. Matuschek, F. Morier-Genoud, U. Keller, V. Scheuer, G. Angelow, and T. Tschudi, "Semiconductor saturable-absorber mirror-assisted Kerr-lens mode-locked Ti:sapphire laser producing pulses in the two-cycle regime," Opt. Lett., vol. 24, pp. 631-633, 1999.

[3] U. Morgner, F. X. Kärtner, S. H. Cho, Y. Chen, H. A. Haus, J. G. Fujimoto, E. P. Ippen, V. Scheuer, G. Angelow, and T. Tschudi, "Sub-twocycle pulses from a Kerr-lens mode-locked Ti:sapphire laser: Addenda," Opt. Lett., vol. 24, p. 920, 1999.

[4] M. Nisoli, S. Stagira, S. D. Silvestri, O. Svelto, S. Sartania, Z. Cheng, M. Lenzner, C. Spielmann, and F. Krausz, "A novel high-energy pulse compression system: Generation of multigigawatt sub-5-fs pulses," Appl. Phys. B, vol. 65, pp. 189-196, 1997.

[5] A. Baltuska, T. Fuji, and T. Kobayashi, "Visible pulse compression to 4 fs by optical parametric amplification and programmable dispersion control," Opt. Lett., vol. 27, pp. 306-308, 2002.

[6] B. Schenkel, J. Biegert, U. Keller, C. Vozzi, M. Nisoli, G. Sansone, S. Stagira, S. De Silvestri, and O. Svelto, "Generation of 3.8-fs pulses from adaptive compression of a cascaded hollow fiber supercontinuum," Opt. Lett., vol. 28, p. 1987, 2003.

[7] H. R. Telle, G. Steinmeyer, A. E. Dunlop, J. Stenger, D. H. Sutter, and U. Keller, "Carrier-envelope offset phase control: A novel concept for asolute optical frequency measurement and ultrashort pulse generation," Appl. Phys. B, vol. 69, pp. 327-332, 1999.

[8] F. W. Helbing, G. Steinmeyer, U. Keller, R. S. Windeler, J. Stenger, and H. R. Telle, "Carrier-envelope offset dynamics of mode-locked lasers," Opt. Lett., vol. 27, pp. 194-196, 2001.

[9] F. W. Helbing, G. Steinmeyer, J. Stenger, H. R. Telle, and U. Keller, "Carrier-envelope-offset dynamics and stabilization of femtosecond pulses," Appl. Phys. B, vol. 74, pp. S35-S42, 2002.

[10] T. Brabec and F. Krausz, "Intense few-cycle laser fields: Frontiers of nonlinear optics," Rev. Mod. Phys., vol. 72, pp. 545-591, 2000.

[11] M. Drescher, M. Hentschel, R. Kienberger, G. Tempea, C. Spielmann, G. A. Reider, P. B. Corkum, and F. Krausz, "X-ray pulses approaching the attosecond frontier," Science, vol. 291, pp. 1923-1927, 2001.

[12] G. G. Paulus, F. Grasborn, H. Walther, P. Villoresi, M. Nisoli, S. Stagira, E. Priori, and S. De Silvestri, "Absolute-phase phenomena in photoionization with few-cycle laser pulses," Nature, vol. 414, pp. 182-4, 2001.

[13] L. Xu, C. Spielmann, A. Poppe, T. Brabec, F. Krausz, and T. W. Hänsch, "Route to phase control of ultrashort light pulses," Opt. Lett., vol. 21, pp. 2008-2010, 1996.

[14] D. J. Jones, S. A. Diddams, J. K. Ranka, A. Stentz, R. S. Windeler, J. L. Hall, and S. T. Cundiff, "Carrier-envelope phase control of femtosecond mode-locked lasers and direct optical frequency synthesis," Science, vol. 288, pp. 635-639, 2000. 
[15] A. Apolonski, A. Poppe, G. Tempea, C. Spielmann, T. Udem, R. Holzwarth, T. W. Hänsch, and F. Krausz, "Controlling the phase evolution of few-cycle light pulses," Phys. Rev. Lett., vol. 85, p. 740, 2000 .

[16] U. Morgner, R. Ell, G. Metzler, T. R. Schibli, F. X. Kärtner, J. G. Fujimoto, H. A. Haus, and E. P. Ippen, "Nonlinear optics with phase-controlled pulses in the sub-two-cycle regime," Phys. Rev. Lett., vol. 86, pp. 5462-5465, 2001.

[17] T. M. Ramond, S. A. Diddams, L. Hollberg, and A. Bartels, "Phase-coherent link from optical to microwave frequencies by means of the broadband continuum from a 1-GHz Ti:sapphire femtosecond oscillator," Opt. Lett., vol. 27, pp. 1842-1844, 2002.

[18] T. Udem, J. Reichert, R. Holzwarth, and T. W. Hänsch, "Accurate measurement of large optical frequency differences with a mode-locked laser," Opt. Lett., vol. 24, pp. 881-883, 1999.

[19] D. v. d. Linde, "Characterization of the noise in continuously operating mode-locked lasers," Appl. Phys. B, vol. 39, pp. 201-217, 1986.

[20] M. J. W. Rodwell, K. J. Weingarten, D. M. Bloom, T. Baer, and B. H. Kolner, "Reduction in the timing fluctuations in a modelocked Nd:YAG laser by electronic feedback," Opt. Lett., vol. 11, pp. 638-640, 1986.

[21] M. J. W. Rodwell, D. M. Bloom, and K. J. Weingarten, "Subpicosecond laser timing stabilization," IEEE J. Quantum Electron., vol. 25, pp. 817-827, 1989

[22] U. Keller, K. D. Li, M. J. W. Rodwell, and D. M. Bloom, "Noise characterization of femtosecond fiber Raman soliton lasers," IEEE J. Quantum Electron., vol. 25, pp. 280-288, 1989.

[23] X. C. Zhang, B. B. Hu, J. T. Darrow, and D. H. Auston, "Generation of femtosecond electromagnetic pulses from semiconductor surfaces," Appl. Phys. Lett., vol. 56, pp. 1011-1013, 1990.

[24] R. A. Kaindl, F. Eickemeyer, M. Woerner, and T. Elsaesser, "Broadband phase-matched difference frequency mixing of femtosecond pulses in GaSe: Experiment and theory," Appl. Phys. Lett., vol. 75, pp. 1060-1062, 1999.

[25] R. Huber, A. Brodschelm, F. Tauser, and A. Leitenstorfer, "Generation and field-resolved detection of femtosecond electromagnetic pulses tunable up to 41 THz," Appl. Phys. Lett., vol. 76, pp. 3191-3193, 2000.

[26] A. Baltuska, T. Fuji, and T. Kobayashi, "Controlling the carrier-envelope phase of ultrashort light pulses with optical parametric amplifiers," Phys. Rev. Lett., p. 133 901, 2002.

[27] M. Mehendale, S. A. Mitchell, J. P. Likforman, D. M. Villeneuve, and P. B. Corkum, "Method for single-shot measurement of the carrier envelope phase of a few-cycle laser pulse," Opt. Lett., vol. 25, pp. 1672-1674, 2000.

[28] M. Kakehata, Y. Fujihira, H. Takada, Y. Kobayashi, K. Torizuka, T. Homma, and $\mathrm{H}$. Takahashi, "Measurements of carrier-envelope phase changes of $100-\mathrm{Hz}$ amplified laser pulses," Appl. Phys. B, vol. 74, pp. S43-S50, 2002.

[29] J. K. Ranka, R. S. Windeler, and A. J. Stentz, "Visible continuum generation in air-silica microstructure optical fibers with anomalous dispersion at $800 \mathrm{~nm}$," Opt. Lett., vol. 25, pp. 25-27, 2000.

[30] M. Nisoli, S. De Silvestri, O. Svelto, R. Szipöcs, K. Ferenz, C. Spielmann, S. Sartania, and F. Krausz, "Compression of high energy laser pulses below 5 fs," Opt. Lett., vol. 22, pp. 522-524, 1997.

[31] U. Keller, C. E. Soccolich, G. Sucha, M. N. Islam, and M. Wegener, "Noise characterization of femtosecond color center lasers," Opt. Lett., vol. 15, pp. 974-976, 1990 .

[32] J. Reichert, R. Holzwarth, T. Udem, and T. W. Hänsch, "Measuring the frequency of light with mode-locked lasers," Opt. Commun., vol. 172, pp. 59-68, 1999 .

[33] K. F. Kwong, D. Yankelevich, K. C. Chu, J. P. Heritage, and A. Dienes, "400-Hz mechanical scanning optical delay-line," Opt. Lett., vol. 18, pp. 558-560, 1993

[34] M. E. Thomas, S. K. Andersson, R. M. Sova, and R. I. Joseph, "Frequency and temperature dependence of the refractive index of sapphire," Infrared Phys. Technol., vol. 39, pp. 235-249, 1998.

[35] G. P. Agrawal, Nonlinear Fiber Optics, 3rd ed. San Diego, CA: Academic, 2001.

[36] R. DeSalvo, A. A. Said, D. J. Hagan, E. W. VanStryland, and M. SheikBahae, "Infrared to ultraviolet measurements of two-photon absorption and $n_{2}$ in wide bandgap solids," IEEE J. Quantum Electron., vol. 32, pp. 1324-1333, 1996.

[37] M. Sheik-Bahae, D. C. Hutchings, D. J. Hagan, and E. W. V. Stryland, "Dispersion of bound electronic nonlinear refraction in solids," IEEE J. Quantum Electron., vol. 27, pp. 1296-1309, 1991.
[38] A. L. Gaeta, "Nonlinear propagation and continuum generation in microstructured optical fibers," Opt. Lett., vol. 27, pp. 924-926, 2001.

[39] M. Takeda, H. Ina, and S. Kobayashi, "Fourier-transform method of fringe-pattern analysis for computer-based topography and interferometry," J. Opt. Soc. Amer. B, vol. 72, pp. 156-160, 1982.

[40] A. Poppe, R. Holzwarth, A. Apolonski, G. Tempea, C. Spielmann, T. W. Hänsch, and F. Krausz, "Few-cycle optical waveform synthesis," Appl. Phys. B, vol. 72, pp. 373-376, 2000.

[41] T. M. Fortier, J. Ye, and S. T. Cundiff, "Nonlinear phase noise generated in air-silica microstructure fiber and its effect on carrier-envelope phase," Opt. Lett., vol. 27, pp. 445-447, 2002.

[42] T. M. Fortier, D. J. Jones, J. Ye, S. T. Cundiff, and R. S. Windeler, "Long-term carrier-envelope phase coherence," Opt. Lett., vol. 27, pp. 1436-1438, 2002.

[43] R. Holzwarth, M. Zimmermann, T. Udem, and T. W. Hänsch, "Optical clockworks and the measurement of laser frequencies with a mode-locked frequency comb," IEEE J. Quantum Electron., vol. 37, pp. 1493-1501, 2001.

[44] S. T. Cundiff, "Phase stabilization of ultrashort optical pulses," J. Phys. D: Appl. Phys., vol. 35, pp. R43-R59, 2002.

[45] S. T. Cundiff, J. Ye, and J.L. Hall, "Optical frequency synthesis based on mode-locked lasers," Rev. Sci. Instrum., vol. 27, pp. 3750-3771, 2001.

[46] A. Bauch and H. R. Telle, "Frequency standards and frequency measurement," Rep. Prog. Phys., vol. 65, pp. 789-843, 2002.

Florian W. Helbing was born in Schwabach, Germany, in 1973. He received the Diploma degree from Erlangen University in 2000. He is working toward the $\mathrm{Ph} . \mathrm{D}$. degree at the Swiss Federal Institute of Technology, Zürich, Switzerland

Mr. Helbing is a member of the Optical Society of America and the German Physical Society.

Günter Steinmeyer (M'96) was born in Bad Pyrmont, Germany, in 1965. He received the Diploma and the Ph.D. degree from Hannover University in 1991 and 1995, respectively. In 2002, he received the Habilitation degree from the Swiss Federal Institute of Technology (ETH) Zürich, Switzerland.

In 1995, he joined the Research Lab of Electronics at MIT, Cambridge, MA, where he worked on ultrafast spectroscopy and photonic bandgap structures. In 1998 , he moved to ETH to work on few-cycle pulse generation, pulse characterization, and chirped mirror design. He recently moved to the Max-Born-Institute, Berlin, Germany.

Dr. Steinmeyer is a member of the Optical Society of America and the German Physical Society.

Ursula Keller (SM'99) was born in Zug, Switzerland, in June 1959. She received the "Diplom" degree in physics from the Swiss Federal Institute of Technology (ETH) Zürich, Switzerland in 1984. She received the M.S. and Ph.D. degrees in applied physics from Stanford University, Stanford, CA, in 1987 and 1989 , respectively.

From late 1984 to 1985 , she worked on optical bistability at Heriot-Watt University, Edinburgh, U.K. For her first year at Stanford she held a Fulbright Fellowship and for the following year she was an IBM Predoctoral Fellow. In 1989, she became a Member of Technical Staff at AT\&T Bell Laboratories, Holmdel, $\mathrm{NJ}$, where she conducted research on photonic switching, ultrafast laser systems, and semiconductor spectroscopy. In March 1993, she was appointed an Associate Professor and in October 1997 she became a Full Professor in the Physics Department at the Swiss Federal Institute of Technology (ETH), Zürich, Switzerland. Her current research interests include ultrafast lasers, attosecond science, ultrafast spectroscopy, and novel devices for applications in optical information processing and communication. She has published more than 190 peer-reviewed journal papers and four book chapters and she holds or has applied for 13 patents.

Dr. Keller received the Carl Zeiss Research Award in 1998 for her pioneering work in novel modelocking and Q-switching techniques using semiconductor saturable absorber mirrors (SESAMs). In 2000, she was elected for the LEOS Distinguished Lecturer Award for modelocked solid-state lasers. She is a fellow of the Optical Society of America (OSA) and a member of the European Physical Society (EPS), the Swiss Physical Society (SPS), and the Swiss Academy of Technical Sciences (SATW). 